Increased EMG intermuscular coherence and reduced signal complexity in Parkinson's disease

Flood, Matthew W; Jensen, Bente Rona; Malling, Anne-Sofie; Lowery, Madeleine M

Published in:

Clinical Neurophysiology

DOI:

10.1016/j.clinph.2018.10.023

Publication date:

2019

Document version

Early version, also known as pre-print

Citation for published version (APA):

Flood, M. W., Jensen, B. R., Malling, A-S., \& Lowery, M. M. (2019). Increased EMG intermuscular coherence and reduced signal complexity in Parkinson's disease. Clinical Neurophysiology, 130(2), 259-269.

https://doi.org/10.1016/j.clinph.2018.10.023 


\title{
INCREASED EMG INTERMUSCULAR COHERENCE AND REDUCED SIGNAL COMPLEXITY IN PARKINSON'S DISEASE
}

\author{
Flood, MW ${ }^{\mathrm{a}, \mathrm{d}^{*}}$, Jensen, $\mathrm{BR}^{\mathrm{b}, \mathrm{c}}$, Malling, $\mathrm{AS}^{\mathrm{b}, \mathrm{c}}$, Lowery, $\mathrm{MM}^{\mathrm{a}, \mathrm{d}^{*}}$ \\ ${ }^{a}$ School of Electrical \& Electronic Engineering, University College Dublin, Belfield, Dublin 4, Ireland \\ ${ }^{b}$ Department of Neurology, Odense University Hospital, University of Southern Denmark, Odense, Denmark \\ 'Integrated Physiology, Dept. of Nutrition, Exercise \& Sports, University of Copenhagen, Copenhagen, \\ Denmark \\ 'Insight Centre for Data Analytics, O’Brien Centre for Science, University College Dublin, Belfield, Dublin 4, \\ Ireland
}

*Corresponding Author: Matthew W. Flood, BSc, ME

Address:

School of Electrical \& Electronic Engineering,

University College Dublin,

Belfield,

Dublin 4,

Ireland.

Email: $\quad$ matthew.flood@ucdconnect.ie

Telephone: $\quad$ +3537161938, +3537161911

Authors: $\quad$ Matthew W. Flood, BSc, ME

School of Electrical \& Electronic Engineering,

University College Dublin, Belfield, Dublin 4, Ireland.

matthew.flood@ucdconnect.ie,matthew.flood@insight-centre.org

Bente Rona Jensen, $\mathrm{PhD}$

Department of Neurology, Odense University Hospital, University of

Southern Denmark, Odense, Denmark

Bente.R.Jensen@rsyd.dk, brjensen@nexs.ku.dk

Anne-Sofie Malling, MSc

Department of Neurology, Odense University Hospital, University of

Southern Denmark, Odense, Denmark

amalling@health.sdu.dk

Madeleine M Lowery, BE, PhD

School of Electrical \& Electronic Engineering, University College Dublin,

Belfield, Dublin 4, Ireland.

madeleine.lowery@ucd.ie

Running Title: Coherence and Complexity of Parkinsonian EMG

Conflict of interest: The authors declare no conflict of interest in the publication of this article. 
Funding:

This work was supported by:

Insight Centre for Data Analytics, Science Foundation Ireland

European Research Council Consolidator Grant (DBSModel 646923)

Odense University Hospital

University of Southern Denmark

Den A. P. Møllerske Støttefond (private foundation)

Merchant L. F. Foghts Foundation (private foundation)

Jascha Foundation (private foundation)

Keywords:

Electromyography, Coherence, Determinism, Entropy, Synchronization, Parkinson's Disease.

Highlights:

- Enhanced MU synchronization in surface EMG during isometric leg extension in Parkinson's disease.

- Higher intermuscular coherence, higher determinism and lower sample entropy in Parkinson's disease.

- Determinism, sample entropy and theta band intermuscular coherence correlate with MDS-UPDRS scores.

Declaration:

All authors have approved the final manuscript for submission. 


\begin{abstract}
:
OBJECTIVES: $\quad$ To investigate differences in surface electromyography (EMG) features in individuals with idiopathic Parkinson's disease (PD) and aged-matched controls.
\end{abstract}

METHODS: $\quad$ Surface EMG was recorded during isometric leg extension in PD patients prior to, and after undergoing a locomotor training programme, and in aged-matched controls. Differences in EMG structure were quantified using determinism (\%DET), sample entropy (SampEn) and intermuscular coherence.

RESULTS: $\quad$ \%DET was significantly higher, and SampEn significantly lower, in PD patients. Intermuscular coherence was also significantly higher in the PD group in theta, alpha and beta frequency bands. \%DET increased and SampEn decreased with increasing Movement-DisorderSociety UPDRS scores, while theta band coherence was significantly correlated with total MDSUPDRS scores and torque variance. Neither \%DET, SampEn nor intermuscular coherence changed in response to training.

CONCLUSIONS: The differences observed are consistent with increased synchrony among motor units within and across leg muscles in PD. Differences between EMG signals recorded from the PD and control groups persisted post-therapy, after improvements in walking capacity occurred. SIGNIFICANCE: These results provide insight into changes in motoneuron activity in PD, demonstrate increased beta band intramuscular coherence in PD for the first time, and support the development of quantitative biomarkers for PD based on advanced surface EMG features. 


\section{INTRODUCTION:}

The severity of the symptoms of Parkinson's disease (PD) are currently clinically evaluated using subjective scores such as the Movement Disorders Society Unified-Parkinson's-Disease-Rating-Scale (MDS-UPDRS) (Goetz et al., 2008). The semi-quantitative score, MDS-UPDRS III, provides a macro view of motor control without characterising specific patient kinematics. There is a recognised need for more quantitative measures to evaluate motor symptoms in PD which can provide insights into changes in neural control and provide an objective biomarker for diagnosis.

Determinism $(\% \mathrm{DET})$ is an effective method of characterising the degree of repeating nonlinear patterns within the EMG signal (Webber et al., 1995; Webber and Zbilut, 1994), and has been shown to capture the progression of muscular fatigue (Webber et al., 1995; Webber and Zbilut, 1994) and short-term motor unit (MU) synchronization better than traditional spectral parameters (Del Santo et al., 2006; Dideriksen et al., 2009; Farina et al., 2002; Fattorini et al., 2005). Conversely, sample entropy (SampEn) is a measure of complexity (Richman and Moorman, 2000), or lack of structure, within a signal. While \%DET and SampEn provide estimates of structure within a given EMG signal, the level of correlation between EMG signals from simultaneously active muscles can be estimated using the coherence between pairs of simultaneously recorded EMG signals, known as intermuscular coherence. Intermuscular coherence can be used to quantify the strength of common, or shared, oscillatory drive to pairs of muscles and has been applied to examine changes across a range of conditions including fatigue, stroke and spinal cord injury (Kattla and Lowery, 2010; Nielsen et al., 2008; Barthélemy et al., 2010). A small number of studies have employed \%DET, SampEn or intermuscular coherence to analyse structural changes in EMG, however, for the most part the applications of these techniques have received little attention in PD to date (Fattorini et al., 2005; Meigal et al., 2009, 2013; van der Stouwe et al., 2015).

Alterations in SampEn and \%DET in PD have been observed and are largely attributed to increased motor unit synchrony associated with rest tremor (Fattorini et al., 2005; Meigal et al., 2009, 2013). Examining wrist and elbow EMG in individuals with different tremor disorders, van der Stouwe et al. (van der Stouwe et al., 2015) have also shown intermuscular coherence to be elevated in PD. 
Parkinsonian tremor is associated with oscillatory activity of central origin, with well-documented correlations between cortical and muscle activity at frequencies linked to Parkinsonian tremor $(4-6 \mathrm{~Hz}$ and 8-10Hz) (Brown, 2003; He et al., 2015; Hurtado et al., 2000; Park et al., 2009). An association between the symptoms of bradykinesia and rigidity and synchronous oscillations within the corticobasal ganglia network has also been identified (Kühn et al., 2009, 2006). Increased beta band (15-30 $\mathrm{Hz}$ ) coherence between oscillations in local field potentials in cortical and subcortical structures has been observed in individuals with PD (Cassidy et al., 2002), with beta band oscillations enhanced in the absence of dopamine and suppressed during deep brain stimulation (Canessa et al., 2016; Kühn et al., 2008). It has been suggested that elevated beta band activity within the cortico-basal ganglia network is antikinetic in nature (Brown, 2003), supported by correlations between changes in beta band power suppression and improvement in bradykinesia and rigidity scores (Kühn et al., 2009).

The presence of elevated levels of synchronous neural activity within the cortico-basal ganglia network in PD could potentially increase synchronization among pre-synaptic inputs to the motoneuron pool, thus influencing motor unit firing patterns. It is not clear, however, whether elevated levels of beta band activity are present in the motoneuron pool and, if so, whether these may contribute to alterations in muscle function. Further investigation of synchronous activity among motor units is thus necessary to understand how changes in motoneuron activity contribute to loss of function in PD. In addition, it remains to be determined whether the alterations in surface EMG properties observed during rest tremor in PD are also present during isometric contraction.

If motor unit synchrony is enhanced within and between muscles in individuals with $\mathrm{PD}$, it may be expected that such alterations in motor unit synchrony could be detected as a reduction in the EMG complexity and an increase in intramuscular coherence in individuals with Parkinson's disease when compared with age-matched controls. The aim of this study was thus to compare surface EMG features related to motor unit synchronization, specifically SampEn, \%DET and intermuscular coherence, between individuals with PD and age-matched controls during isometric contraction. EMG signals were recorded from quadriceps and hamstring muscles during submaximal isometric leg extension, distinguishing this study from previous studies which have examined EMG features in the 
upper limb during parkinsonian tremor (Fattorini et al., 2005; Meigal et al., 2009, 2013; Scholten et al., 2016; Vaillancourt and Newell, 2000).

EMG features were also compared in the PD group, prior to and post participation in an anti-gravity, bodyweight-supported, high-intensity locomotor training programme which has previously been reported to benefit patients with $\mathrm{PD}$, to investigate whether the improvements in motor function were accompanied by a change in EMG signal properties. A reduction in total MDS-UPDRS score and improvement in motor outcomes with this training was previously observed (Rose et al., 2013a; Rose et al., 2013c). The present study further investigates whether these changes were accompanied by alterations in EMG features which could indicate potential neuromuscular adaptations that may underlie the benefits observed. 


\section{METHODS}

\subsection{Study Participants}

Thirteen male patients ( $63 \pm 6$ yrs) with idiopathic Parkinson's were recruited by a neurologist at the Bispebjerg University Hospital, Copenhagen, Denmark. Subjects included in the study were diagnosed with PD according to the UK Parkinson's Disease Society Brain Bank Criteria, and each subject had an effective response to parkinsonian medication. Exclusion criteria included any known disease (other than PD) which may influence motor function, any contraindications to exertion during physical exercise, and patients treated for PD through deep brain stimulation. Each PD subject underwent an 8 week high-intensity locomotor therapy involving a positive pressure anti-gravity treadmill (Rose et al., 2013a), and were assessed at three stages - 8 weeks pre-training (control period), immediately prior to intervention initiation, and after the intervention ( 8 weeks later). At each stage, patient performance was assessed using MDS-UPDRS scores and isometric leg extension tasks. All PD subjects followed their routine medication schedule and performed all experimental procedures during the self-declared ON state (Rose et al., 2013a). Each participant conducted the tests at the same time of day in order to reduce the eventual influence of intra-day motor fluctuations, and MDS-UPDRS scores were evaluated on a separate day to the experiment. Due to an illness he acquired unrelated to Parkinson's disease, one subject was unable to complete all post-training tests. The data from all 13 PD subjects was included from both pre-training stages, whereas only data from the 12 subjects who completed the post-training tests was analysed in the post-training stage. For further clinical information regarding the PD subjects see Table 1.

Thirteen healthy age-matched male controls $(65 \pm 5 \mathrm{yrs})$ performed the same isometric leg extension task. Control subjects were assessed for exclusion criteria, including diseases influencing motor function. This study was conducted according to the principles of the Declaration of Helsinki and approved by the Committee on Ethics in Science in Copenhagen (H-3-2010-051). For further information regarding the study participants and experimental protocol, see Rose et al. (Rose et al., 2013b). 


\subsection{Experimental Protocol \& Data Acquisition}

Subjects performed an isometric leg extension at $15 \%$ of their maximum voluntary contraction against a resistive load applied at the ankle. On-line visual force feedback was provided on a computer screen placed $1 \mathrm{~m}$ away at eye level. Isometric contractions were chosen to reduce the contribution of rest tremor and mechanical-reflex oscillations on the surface EMG signal. Isometric contractions also enable surface EMG features related to motor unit activity and synchronization to be examined without the confounding effects of factors such as changes in muscle length, soft-tissue geometry and the electrode position which occur during dynamic contraction.

Each test had four trials per leg consisting of a contraction lasting $25 \mathrm{~s}$ (Fig. 1A), separated by a $65 \mathrm{~s}$ rest to limit the effect of fatigue. Surface EMG (Logger Technology, Sweden; 10-400Hz) was recorded with bipolar electrodes from the five muscles of the upper leg - vastus medialis (VM), vastus lateralis (VL), rectus femoris (RF), semitendinosus (ST), and biceps femoris (BF). After skin preparation, bipolar electrodes (Ambu ${ }^{\circledR}$ Neuroline 720, Ballerup, Denmark) were placed with a $20 \mathrm{~mm}$ inter-electrode distance above the muscle bellies of the knee extensor and flexor muscles according to Perotto (Perotto, 2005). Surface EMG was AD-converted at 1kHz (DT 9834, Data Translation, Marlboro, MA, USA) and later visually inspected in MatLab (Mathworks, Natick, MA, USA). The variance of the torque signal was estimated as a measure of the steadiness of the contractile force exerted by the subject.

Total MDS-UPDRS score and MDS-UPDRS III motor scores were evaluated by the same movement disorder specialist at each stage of therapy.

\subsection{Data Analysis}

\subsubsection{Determinism (\%DET)}

Recurrence Quantification Analysis (RQA) is a nonlinear mathematical method that includes a family of measures used to explain dynamical systems such as EMG, which are composed of many contributing parts. The first step in RQA is to create a series of vectors from $m$ number of samples of the signal spaced a defined length apart, $\lambda$. The Euclidean distance between all pairs of vectors is then 
calculated to represent a phase space. A threshold is selected which determines the minimum tolerance within which recurring patterns must match to constitute a recurring similarity. A binary recurrence plot is then constructed (Fig. 1D), which indicates the combinations of points which fall within the tolerance of each other. Finally, the information provided by the recurrence plot is quantified through the calculation of quantification variables including the percentage recurrence (\%REC), which is the density of points in the recurrence plot, and the percentage determinism (\%DET), which is the number of points in diagonal lines of a given length. \%REC represents the number of repeating patterns in the signal, whereas $\% \mathrm{DET}$ is a measure of the number patterns that repeat continuously over a period of time. \%REC and \%DET thus represent the incidence of nonlinear patterns and the degree of nonlinear structure over a given time period respectively.

The RQA parameters selected in this study (Table 2) were chosen to capture the dynamics the observed system, i.e. motor unit firing patterns (Marwan et al., 2007). RQA was performed on five non-overlapping $1.5 \mathrm{~s}$ segments of EMG in each trial. The firing activity of motor units can be assumed to be statistically invariant over a period of approximately $1.5 \mathrm{~s}$, thus a window length of 1.5 $\mathrm{s}$ was chosen to preserve this stationarity property. $\% \mathrm{DET}$ was calculated from the recurrence matrix using the parameters provided in Table 2. \%DET values were averaged across the five segments in each trial. The equations used to calculate the \%REC and \%DET are provided in the Appendix.

\subsubsection{Sample Entropy (SampEn)}

Entropy is a method of quantifying the degree of regularity in signals in the presence of deterministic chaotic and random processes. In this study, we have chosen to quantify the entropy of the EMG signal using sample entropy (SampEn), a measure of complexity and regularity which has been derived specifically for physiological time series and which avoids the limitations of alternative approaches (Richman and Moorman, 2000). SampEn involves a template matching process, where a template vector, an EMG segment of $m$ samples, is compared to all other sequential templates in the signal. A match occurs when the difference between two templates is within a defined tolerance, $r$. This process is repeated for templates of length $m+1$, and the ratio of total template matches is used to estimate SampEn. SampEn in each trial was estimated from five overlapping $1.5 \mathrm{~s}$ segments of 
EMG using the parameters provided in Table 2. The equations for calculating the sample entropy are provided in the Appendix.

\subsubsection{Intermuscular Coherence}

Magnitude squared coherence, $C_{x y}(f)$, is a measure of the correlation between two signals as a function of frequency, $f$, defined as

$$
C_{x y}=\frac{\left|S_{x y}(f)\right|^{2}}{S_{x x}(f) S_{y y}(f)}
$$

where $S_{x y}(f)$ is the cross spectrum and $S_{x x}(f)$ and $S_{y y}(f)$ are the auto spectra of $x(t)$ and $y(t)$ respectively (Bendat and Piersol, 1971). The magnitude squared coherence between EMG signals recorded from two muscles, known as intermuscular coherence, provides a measure of the correlation between the signals as a function of frequency. Intermuscular coherence between agonist-agonist and agonist-antagonist muscle pairs was estimated using Welch's averaged modified periodogram method using the parameter values provided in Table 2. Antagonist-antagonist coherence was excluded from the analysis due to crosstalk evident by consistently high levels of coherence across all frequencies. Intermuscular coherence in each leg was calculated from rectified EMG signals, concatenated from all four trials. Coherence was considered significant at each frequency $(\alpha<0.05)$ if higher than a threshold, $Z$, as described by Terry and Griffin (Terry and Griffin, 2008). Intermuscular coherence in each frequency band, theta $(4-8 \mathrm{~Hz})$, alpha $(8-12 \mathrm{~Hz})$, beta $(12-30 \mathrm{~Hz})$, gamma low $(30-60 \mathrm{~Hz})$ was estimated as the integral of coherence above $Z$.

\subsubsection{Statistical Analysis}

The value of the nonlinear features (\%DET \& SampEn) at each stage of therapy was estimated for each subject as the average from all four trials in each leg. Statistical analysis of nonlinear EMG features was restricted to the most affected leg in PD subjects, whereas the average from both legs was calculated for control subjects. No significant difference was observed between EMG features on the more and less-affected sides. The Anderson-Darling test indicated that the results of features were non-normally distributed, thus subsequent tests for significance between the PD and control groups 
were performed using the Wilcoxon Rank-Sum test. Within group comparisons of EMG features pre and post therapy were performed using a paired Wilcoxon Signed Rank test. A 2 - degree Bonferroni Correction was applied to adjust for multiple comparisons between the control group and the PD group, pre and post therapy.

Both pre-training stages, henceforth referred to as Pre1 and Pre2, functioned as control periods against which the effect of therapy was assessed. Pre1 and Pre2 were averaged when performing statistical tests between PD and control subjects to obtain the same number of trials in both groups for comparison. Correlations between EMG features and MDS-UPDRS scores at each stage were calculated using Spearman's rank correlation. The corrected alpha level for multiple correlations assuming independent tests was 0.0102 (Curtin and Schulz, 1998). In cases where muscles were examined according to group (agonist or antagonist), \%DET and SampEn values were averaged across agonist (VM, VL, RF) and antagonist (BF, ST) muscle groups.

High levels of crosstalk, evidenced by unusually high coherence across all frequencies, was observed between the vastus medialis and rectus femoris muscles of subject 13, post-training. These data were therefore omitted from the analysis and the average agonist-agonist intermuscular coherence posttherapy included only 11 of the 13 subjects. All EMG signal features and statistical tests were estimated using custom developed algorithms using MatLab 2015a (MathWorks, Natick, MA, USA). 


\section{RESULTS}

No significant difference in maximal voluntary contractile torque during isometric extension was observed between the control (235.67 Nm \pm 61.03 ) and PD groups (Pre1: 209.35 Nm \pm 58.61 ; Pre2: 203.06 $\mathrm{Nm} \pm 54.31$; Post: $224.47 \mathrm{Nm} \pm 53.11)$ at any stages. Torque variability was significantly higher in the PD patients compared with controls (coefficient of variation - PD: $2.02 \pm 0.91$, Controls: $1.47 \pm 0.36, \mathrm{p}=0.02)$. The frequency at which maximum power occurred in the power spectrum of the torque signal was $4.42 \mathrm{~Hz} \pm 0.90$ in the $\mathrm{PD}$ group, and $4.3 \mathrm{~Hz} \pm 1.19$ in the control group $(\mathrm{p}=0.79)$.

\subsection{Determinism (\%DET)}

Median values of \%DET in the most affected leg were consistently higher in the PD group in both agonist and antagonist muscles pre and post training, Fig. 2. Significant differences were observed between mean \%DET of patients and controls in the BF muscle, Table 3. When averaged over agonist and antagonist muscle groups, \%DET was higher in the patient group and significantly higher in the antagonist muscles of the individuals with PD post-training, Fig. 3B (antagonists - Post: $\mathrm{p}=$ $0.005)$.

\subsection{Sample Entropy (SampEn)}

Median SampEn values were consistently lower in EMG of PD subjects at both stages of training, with significant differences again observed in the BF muscle, Table 3. When averaged across muscle groups, significant differences between the subject groups were observed in agonist and antagonist muscles post training Fig. $3 \mathrm{C}-3 \mathrm{D}$, (agonists - Post: $\mathrm{p}=0.021$; antagonists - Pre: $\mathrm{p}=0.012$; Post: $\mathrm{p}=$ $0.003)$.

When comparing agonist and antagonist muscles within subject groups, \%DET was significantly higher and SampEn was significantly lower in the agonist muscle group for both controls and PD subjects at all training stages $(\mathrm{p}<0.001)$. 


\subsection{Intermuscular Coherence}

Average intermuscular coherence between agonist muscle pairs was higher in the PD group than controls in each frequency band examined (Fig. 4). Intermuscular coherence in PD was significantly higher in the alpha band $(8-12 \mathrm{~Hz})$ pre training and beta $(12-30 \mathrm{~Hz})$ band pre and post training (Fig. 4). Similarly, average intermuscular coherence between agonist-antagonist muscle pairs was higher in the PD group, with significant differences observed between subject groups in the beta band post-training $(\mathrm{p}=0.014)$.

\subsection{Correlation with MDS-UPDRS}

The correlation between \%DET and SampEn and MDS-UPDRS motor and total scores is presented in Fig. 5. A positive correlation was observed between the \%DET and MDS-UPDRS motor and total scores in Pre1 and Post training stages (Fig. 5A). Consistent with this, an inverse correlation between MDS-UPDRS and SampEn was observed at Pre1 and Post stages (Fig. 5B). Though a consistent trend of increasing \%DET and decreased SampEn with increasing MDS-UPDRS scores was observed, the significance level did not always reach the lower level for statistical significance when alpha was corrected for multiple comparisons.

Theta band (4-8Hz) agonist-agonist intermuscular coherence and total MDS-UPDRS scores were positively correlated at Pre1 $(r=0.58, \mathrm{p}=0.040)$, Pre2 $(\mathrm{r}=0.60, \mathrm{p}=0.030)$ and Post $(\mathrm{r}=0.80, \mathrm{p}=$ 0.003) training stages (Fig. 5C). Theta band intermuscular coherence was also significantly correlated with the coefficient of variation of torque exerted during the isometric leg extension task pre $(r=0.57, p=0.043)$ and post training $(r=0.82 \mathrm{p}=0.004)($ Fig. 5D). Although positive correlations were observed between MDS-UPDRS scores and intermuscular coherence in every other frequency band examined at each stage of training, no consistently significant relationship was observed.

No correlation was observed between any EMG feature and the Levodopa equivalent dosage, and no effect of training was observed on any of the EMG features examined. 


\section{DISCUSSION:}

Higher determinism, lower sample entropy and higher theta, alpha and beta band intermuscular coherence were observed in the surface EMG of hamstring and quadriceps muscles of individuals with Parkinson's disease during submaximal isometric leg extension when compared with agematched controls. Collectively these differences suggest that motor unit synchronization, both within and between muscles, is elevated in the leg muscles in Parkinson's disease. Furthermore, determinism, sample entropy and theta band coherence were found to be correlated with patient MDSUPDRS scores.

\%DET was higher and SampEn was significantly lower in the PD group than in controls pre and post training (Fig. 3 and Table 3). The results demonstrate the complementary effect of both features in quantifying the degree of structure (or complexity) within the surface EMG signal, where differences in \%DET and SampEn observed in the PD group indicate the presence of enhanced deterministic behaviour in Parkinsonian EMG. A subsidiary result found that \%DET and SampEn were significantly higher and lower, respectively, in the agonist muscles than in the antagonist muscles in both PD and control groups ( $\mathrm{p}<0.001)$, Fig. 3 .

In individuals with PD, Fattorini et al. (Fattorini et al., 2005), observed a reduction in EMG \%DET during isometric voluntary contraction, when compared with rest conditions, attributed to higher levels of motor unit synchronization during to rest tremor. In the present study, we examined surface EMG activity recorded under normalized isometric loading conditions. The differences in \%DET, SampEn and coherence observed here thus likely reflect altered patterns of motor unit activity in PD, different to those associated with parkinsonian rest tremor which is typically accompanied by clear bursting of the EMG signal (Glendinning and Enoka, 1994) not present in the isometric contractions examined here, (see for example Fig. 1A). \%DET has previously been associated with motor unit synchronization occurring during isometric muscle fatigue (Farina et al., 2002; Webber et al., 1995), with simulation studies demonstrating a correlation between \%DET and the level of synchronization (Fattorini et al., 2005). Direct comparison of surface EMG determinism and short-term motor unit 
synchronization from experimentally recorded data have indicated a more complex relationship and the influence of additional factors (Dideriksen et al., 2009; Schmied and Descarreaux, 2011).

Meigal et al. (Meigal et al., 2009) have similarly observed higher \%DET and lower SampEn in PD patients when compared with old control subjects under loading of the upper limbs. However, differences in \%DET between PD and old control subjects were only significant in the unloaded condition, and though SampEn was lower in the PD group than older control subjects, it was not significantly so in their study (Meigal et al., 2009).

In addition to the changes in SampEn and \%DET, intermuscular coherence between agonist-agonist and agonist-antagonist pairs was higher in the PD group in the theta, alpha and beta bands, pre- and post-training (Fig. 4). The effect of PD on agonist-agonist intermuscular coherence was estimated using the Cohen's $-\mathrm{d}$ statistic. Despite the high inter-subject variability associated with intermuscular coherence, PD was considered to have a large effect on intermuscular coherence across all frequency bands examined, pre and post training, indicated by $d$ values ranging from 0.69-1.23.

Enhanced theta and alpha band intermuscular coherence may reflect increased oscillatory drive in the 4-6 Hz and 8-12 Hz frequency ranges to the spinal motoneuron pool, hypothesized to drive tremor in PD (Timmermann et al., 2003). The findings are also consistent with those of van der Stouwe et al. (van der Stouwe et al., 2015) who reported high coherence at the tremor frequency in PD patients in the upper limb, though in that study tremor was clearly present as evidenced by bursting patterns in the EMG signal not present during the loaded isometric contractions examined here.

Beta band coherence, similar to short-time motor unit synchronization, is generally assumed to be cortical in origin (Conway et al. 1995). In the context of PD, synchrony of beta band oscillations in the STN and basal ganglia network has been proposed to be anti-kinetic, having been associated with the symptoms of bradykinesia and rigidity (Kühn et al., 2009, 2006), and shown to reduce with both levodopa and during deep brain stimulation. The significance of alterations in beta band coherence in the peripheral nervous system in PD, however, is not clear. In contrast to the increase in beta-band intermuscular coherence observed here, reduced beta band intermuscular coherence has previously 
been observed in PD patients (Marsden et al., 2001). However, in that study a relatively high cut-off frequency was employed for the lower edge of the EMG band-pass filter (56-300 Hz) (Marsden et al., 2001), which could alter the information available. Additionally, the contraction type may influence the coherence observed, with isometric contractions of the lower limb investigated in the present study in contrast with wrist flexion examined by Marsden et al., 2001.

Given the association between motor unit synchronization and cortical inputs (Conway et al., 1995), and observed increases in theta and beta band synchronous neural activity in the cortico-basal ganglia network in PD (Kühn et al., 2009), it is possible that the increased \%DET, reduced SampEn and elevated theta, alpha and beta coherence in PD may reflect greater oscillatory neural synchrony within the central nervous system that is transmitted to the motoneuron pool, resulting in enhanced motor unit synchrony. Alternatively, it may be the result of enhanced reflex activity in peripheral feedback circuits, changes within the muscle or a combination of these factors. There is evidence of motoneuron degeneration in idiopathic PD, leading to motoneuron atrophy and reinnervation, with a resulting reduction in the total number of MU's and larger motor unit action potentials (Caviness et al., 2002, 2000). These physiological adaptations could result in sparser EMG signals dominated by larger action potentials, giving rise to a more structured, less complex EMG.

No effect of training was observed on any of the EMG features examined in the present study. In the same patient population, Rose et al. reported significant improvements in patient MDS-UPDRS scores and walking capacity in response to high-intensity locomotor therapy (Rose et al., 2013a). The absence of significant changes in the EMG features examined here, in the context of improvements in walking capacity, could be accounted for by motor adaptations such as intralimb coordination, alterations in motor unit recruitment or rate coding, which are not captured in the EMG signal features examined. The results further suggest that the observed improvements in patient outcomes are not related to a reduction of motor unit synchrony, which remained higher in the patient group posttraining.

The correlations between MDS-UPDRS scores and both \%DET and SampEn observed in the present study (Fig. 5) are consistent with, and extend the findings reported by Meigal et al. (Meigal et al., 
2009). Theta band $(4-8 \mathrm{~Hz})$ coherence covers the frequency range which contributes most strongly to Parkinsonian tremor. As such, it was hypothesized that force variability should reflect tremor and correlate with theta band intermuscular coherence. Consistent with this, a significant correlation was observed between theta band intermuscular coherence and the coefficient of variation of torque in Pre2 and Post (Fig. 5).

The MDS-UPDRS total scores tended to exhibit a stronger correlation with the \%DET and SampEn than the MDS-UPDRS motor scores alone, Fig. 5. One possibility is that the UPDRS total scores, incorporating both Mentation, Behaviour \& Mood in addition to Motor Examination, capture additional factors such as stress and anxiety which can have a profound influence on parkinsonian symptoms, particularly relating to freezing of gait (Giladi and Hausdorff, 2006, Martens et al., 2014).

\subsection{Methodological Considerations}

Recurrence quantification analysis is very sensitive to the choice of input parameters, especially when applied to physiological data where noise and stationarity are prominent issues. RQA parameters are typically based on studies of well-defined chaotic systems where the deterministic structure is strongly evident. The application of RQA to EMG, which is quasi-stationary and has high intra and inter-subject variability, is thus limited in its capacity to accurately depict the underlying dynamics of the neuromuscular system. This limitation may contribute to the relatively high inter-subject variability observed, and the finding that although \%DET was consistently higher in the PD group in each muscle examined, it did not reach significance in agonists when averaged across muscle groups.

SampEn was developed to correct two deficits of approximate entropy (ApEn) by removing the selfmatching bias in its calculation, and improving robustness to short data sets (Richman and Moorman, 2000). However, like RQA, SampEn is still subject to high variability and precise parameter selection. Furthermore, it has been documented that increased entropy does not always reflect increased dynamical complexity, and may change over different scales (Costa et al., 2002).

Finally, the effect of medication as a confounding effect on the results of this study cannot be excluded. Though no relationship between Levodopa equivalent dosage and any of the EMG features 
examined was observed, studies have consistently reported a dopamine-dependent modulation of oscillatory activity within the cortico-basal-ganglia network, including a reduction in beta band oscillations with Levodopa and increase in high gamma oscillatory activity (Kuhn et al., 2006; Kuhn et al., 2017).

\section{CONCLUSIONS:}

Differences were observed in nonlinear surface EMG features and intermuscular coherence between individuals with PD and age-matched controls during submaximal isometric contraction. The results provide evidence for the first time of elevated beta-band intermuscular coherence in PD. Differences between the control and PD groups were present both before and after high intensity locomotor training which improved walking capacity. Determinism, sample entropy, and theta band intermuscular coherence were significantly correlated with patient MDS-UPDRS scores. Collectively, the differences in \%DET, SampEn and intermuscular coherence observed suggest elevated levels of motor unit synchrony, both within individual muscles and between simultaneously active muscles in Parkinson's disease during isometric contraction, though changes in motor unit morphology including motoneuron degeneration may also contribute. 


\section{APPENDIX}

Estimation of Determinism (\%DET) and Sample Entropy (SampEn)

Recurrence Quantification Analysis graphically represents nonlinear changes in system dynamics (Eckmann et al., 1987). Using Taken's theorem (Takens, 1981), an $m$ dimensional phase space is reconstructed from a one dimensional signal through $N$ multidimensional time delayed state vectors, $x_{i}$, which here represent the successive samples of the EMG signal separated by $\lambda$ samples (Eckmann et al., 1987; Marwan et al., 2007; Takens, 1981). A Euclidean distance matrix, $\mathbf{R}_{i, j}$, is created from the phase space and transformed into a recurrence plot (RP) as follows:

$$
\mathbf{R}_{i, j}(\varepsilon)=\Theta\left(\varepsilon-\left\|\vec{x}_{i}-\vec{x}_{j}\right\|\right), \quad i, j=1, \ldots, N, \quad \text { Eq. (A.1) }
$$

where $i, j$ represent the row and column index respectively, $\varepsilon$ is a threshold distance, and $\Theta$ is the Heaviside function. If points in the phase space are within a radius of size $\varepsilon$, a black dot is placed in the RP. After distances between all points have been transformed, the binary recurrence plot is complete (Fig. 1D), where black dots represent states in the system exhibiting recurrent behaviour.

The recurrence rate (\%REC) (Eq. A.2) and percentage determinism (\%DET) (Eq. A.3) are calculated from the RP using

$$
\begin{aligned}
& { }_{0 R E C_{(\varepsilon)}}=\frac{1}{N^{2}} \sum_{i, j=1}^{N} \mathbf{R}_{i, j}(\varepsilon) \\
& { }_{0 E T_{(l, \varepsilon)}}=\frac{\sum_{l=l_{\min }}^{N} l . P(l)}{\sum_{l=1}^{N} l . P(l)}
\end{aligned}
$$

where $P(l)$ is the histogram of points forming diagonal lines in the RP greater than or equal to a specified minimum line length, $l_{\min }$ \% REC is a measure of the density of the RP, a commensurate measure to the correlation sum $-C_{2}(\varepsilon)$ (Grassberger and Procaccia, 1983; Marwan et al., 2007).

In calculating SampEn, the distance, $d$, between $N-m$ vectors, $x_{m}$, composed of sequences of $m$ points, are matched throughout the signal, $u$, by Eq.'s A.4.

$$
d\left[x_{m}(i), x_{m}(j)\right]=\max \{|u(i+k)-u(j+k)|: 0 \leq k \leq m-1\}, \quad i, j,(i \neq j)=1, \ldots, N-m,
$$




$$
\begin{gathered}
B_{m}^{i}(r)=\frac{1}{N-m} \sum_{i \neq j}^{N-m} d\left[x_{m}(i), x_{m}(j)\right] \leq r, \\
A_{m}^{i}(r)=\frac{1}{N-m} \sum_{i \neq j}^{N-m} d\left[x_{m+1}(i), x_{m+1}(j)\right] \leq r, \quad \text { (Eq.'s A.4) }
\end{gathered}
$$

SampEn is then defined as the conditional probability that vectors of $m$ points within a tolerance $r$, $B_{m}(r)$, will also match for vector lengths of $m+1, A_{m}(r)$, for signal of $N$ samples long (Eq. A.5).

$$
\operatorname{SampEn}(m, r, N)=-\ln \frac{A_{m}(r)}{B_{m}(r)} \quad \text { (Eq. A.5) }
$$

All parameter values used to calculate the SampEn, \%DET and intermuscular coherence are given in Table 2. 


\section{FINANCIAL DISCLOSURES:}

M.W.F. and M.M.L.:

Insight Centre for Data Analytics, Science Foundation Ireland

European Research Council Consolidator Grant (DBSModel 646923)

B.R.J. and A.S.M.:

Odense University Hospital

University of Southern Denmark

Den A. P. Møllerske Støttefond (private foundation)

Merchant L. F. Foghts Foundation (private foundation)

Jascha Foundation (private foundation) 


\section{REFERENCES}

Barthélemy D, Willerslev-Olsen M, Lundell H, Conway BA, Knudsen H, Biering-Sørensen F, et al. "Impaired transmission in the corticospinal tract and gait disability in spinal cord injured persons." J. Neurophysiol. 2010; 104: 1167-1176. doi:10.1152/jn.00382.2010

Bendat JS, Piersol AG, Random Data: Analysis and Measurement Procedures, 1971.

Brown P, "Oscillatory Nature of Human Basal Ganglia Activity: Relationship to the Pathophysiology of Parkinson's Disease." Movement Disord. 2003; 18: 357-63. doi:10.1002/mds. 10358 .

Canessa A, Pozzi NG, Arnulfo G, Brumberg J, Reich MM, Pezzoli G, et al., "Striatal Dopaminergic Innervation Regulates Subthalamic Beta-Oscillations and Cortical-Subcortical Coupling during Movements: Preliminary Evidence in Subjects with Parkinson's Disease.” Front. Hum. Neurosci. 2016; 10: 611. doi:10.3389/fnhum.2016.00611.

Cassidy M, Mazzone P, Oliviero A, Insola A, Tonali P, Di Lazzaro V, et al., "Movement-Related Changes in Synchronization in the Human Basal Ganglia." Brain 2002; 125: 1235-46 doi:10.1093/brain/awf135.

Caviness JN, Smith BE, Clarke Stevens J, Adler CH, Caselli RJ, Hentz JG, et al., "Motor Unit Number Estimates in Idiopathic Parkinson's Disease.” Parkinsonism Relat Disord. 2002; 8: $161-64$. doi:10.1016/S1353-8020(01)00007-4.

Caviness JN, Smith BE, Clarke Stevens J, Adler CH, Caselli RJ, Reiners CA, et al., "Motor Unit Changes in Sporadic Idiopathic Parkinson's Disease.” Movement Disord. 2000; 15: 238-43. doi:10.1002/1531-8257(200003)15:2<238::AID-MDS1006>3.0.CO;2-J.

Conway BA, Halliday DM, Farmer SF, Shahani U, Maas P, Weir AI, et al., "Synchronization between Motor Cortex and Spinal Motoneuronal Pool during the Performance of a Maintained Motor Task in Man.” J. Physiol. 1995; 489: 917-24. 
doi:10.1113/jphysiol.1995.sp021104.

Costa M, Goldberger AL, Peng CK, "Multiscale Entropy Analysis of Complex Physiologic Time Series." Phys. Rev. Lett. 2002; 89: 68102.

doi:10.1103/PhysRevLett.89.068102.

Curtin F, Schulz P, “Multiple correlations and Bonferroni’s correction.” Biol. Psychiat, 1998; 44: 775-777.

doi:10.1016/S0006-3223(98)00043-2

Del Santo F, Gelli F, Schmied A, Vedel JP, Rossi A, Mazzocchio R, "Motor Unit Synchronous Firing as Revealed by Determinism of Surface Myoelectric Signal." J. Neurosci. Meth. 2006; 155: $116-21$. doi:10.1016/j.jneumeth.2006.01.003.

Dideriksen JL, Falla D, Baekgaard M, Mogensen ML, Steimle KL, Farina D, “Comparison between the Degree of Motor Unit Short-Term Synchronization and Recurrence Quantification Analysis of the Surface EMG in Two Human Muscles." Clin. Neurophysiol. 2009; 120: 208692.

doi:10.1016/j.clinph.2009.09.011.

Eckmann JP, Oliffson Kamphorst S, Ruelle D, “Recurrence Plots of Dynamical Systems.” EPL-Europhys. Lett. 1987; 4: 973-77. doi:10.1209/0295-5075/4/9/004

Farina D, Fattorini L, Felici F, Filligoi G, "Nonlinear Surface EMG Analysis to Detect Changes of Motor Unit Conduction Velocity and Synchronization.” J. Appl. Physiol. 2002; 93: 1753-63. doi:10.1152/japplphysiol.00314.2002.

Fattorini L, Felici F, Filligoi G, Traballesi M, Farina D, "Influence of High Motor Unit Synchronization Levels on Non-Linear and Spectral Variables of the Surface EMG.” J. Neurosci. Meth. 2005; 143: 133-39. doi:10.1016/j.jneumeth.2004.09.018.

Glendinning DS, Enoka RM, "Motor unit behavior in Parkinson's disease." Phys. Ther. 1994; 
74: 61-70.

doi:10.1093/ptj/74.1.61

Giladi N, Hausdorff J, "The role of mental function in the pathogenesis of freezing of gait in Parkinson's disease.” J. Neurol. Sci. 2006; 248: 173-176. doi:/10.1016/j.jns.2006.05.015

Goetz CG, Tilley BC, Shaftman SR, Stebbins GT, Fahn S, Martinez-Martin P, et al., "Movement Disorder Society-Sponsored Revision of the Unified Parkinson's Disease Rating Scale (MDSUPDRS): Scale Presentation and Clinimetric Testing Results.” Movement Disord. 2008; 23: 2129-70. doi:10.1002/mds.22340.

Grassberger P, Procaccia I, “Characterization of Strange Attractors.” Phys. Rev. Lett. 1983; 50: $346-49$. doi:10.1103/PhysRevLett.50.346.

Hurtado JM, Lachaux JP, Beckley DJ, Gray CM, Sigvardt KA, "Inter- and Intralimb Oscillator Coupling in Parkinsonian Tremor." Movement Disord. 2000; 15: 683-91. http://www.ncbi.nlm.nih.gov/pubmed/10928579.

Kattla S, Lowery M, "Fatigue related changes in electromyographic coherence between synergistic hand muscles." Exp. Brain Res. 2010; 202: 89-99. doi:10.1007/s00221-009-2110-0

Kühn AA, Kempf F, Brücke C, Gaynor Doyle L, Martinez-Torres I, Pogosyan A, et al., "HighFrequency Stimulation of the Subthalamic Nucleus Suppresses Oscillatory $\beta$ Activity in Patients with Parkinson's Disease in Parallel with Improvement in Motor Performance.” $J$. Neurosci. 2008; 28: 6165-6173 doi:10.1523/JNEUROSCI.0282-08.2008

Kühn AA, Kupsch A, Schneider GH, Brown P, "Reduction in Subthalamic 8-35 Hz Oscillatory Activity Correlates with Clinical Improvement in Parkinson's Disease.” Eur. J. Neurosci. 2006; 23: 1956-60.

doi:10.1111/j.1460-9568.2006.04717.x. 
Kühn AA, Tsui A, Aziz T, Ray N, Brücke C, Kupsch A, et al., "Pathological Synchronisation in the Subthalamic Nucleus of Patients with Parkinson's Disease Relates to Both Bradykinesia and Rigidity." Exp. Neurol. 2009; 215: 380-87. doi:10.1016/j.expneurol.2008.11.008.

Marsden J, Limousin-Dowsey P, Fraix V, Pollak P, Odin P, Brown P. “Intermuscular Coherence in Parkinson's Disease: Effects of Subthalamic Nucleus Stimulation.” Neuroreport 2001; 12: $1113-17$.

doi:10.1097/00001756-200105080-00013

Martens K, Ehgoetz A, Ellard CG, Almeida QJ, "Does anxiety cause freezing of gait in Parkinson's disease?", PLoS One 2014; 9: e106561. doi:10.1371/journal.pone.0106561

Marwan N, Romano MC, Thiel M, Kurths J, "Recurrence Plots for the Analysis of Complex Systems.” Phys. Rep. 2007; 438: 237-329. doi:10.1016/j.physrep.2006.11.001.

Meigal AI, Rissanen S, Tarvainen MP, Karjalainen PA, Iudina-Vassel IA, Airaksinen O, et al., "Novel Parameters of Surface EMG in Patients with Parkinson's Disease and Healthy Young and Old Controls." J. Electromyogr Kinesiol. 2009; 19: e206-213. doi:10.1016/j.jelekin.2008.02.008.

Meigal AI, Rissanen S, Tarvainen MP, Airaksinen O, Kankaanpää M, Karjalainen PA, "Non-Linear EMG Parameters for Differential and Early Diagnostics of Parkinson's Disease." Front. Neurol. 2013; 4: 135. doi:10.3389/fneur.2013.00135.

Nielsen JB, Brittain JS, Halliday DM, Marchand-Pauvert V, Mazevet D, Conway BA. "Reduction of common motoneuronal drive on the affected side during walking in hemiplegic stroke patients." Clin. Neurophysiol. 2008; 119: 2813-2818. doi:org/10.1016/j.clinph.2008.07.283

Park H, Kim JS, Paek SH, Jeon BS, Lee JY, Chung CK, “Cortico-Muscular Coherence Increases with 
Tremor Improvement after Deep Brain Stimulation in Parkinson's Disease.” NeuroReport 2009; 20: 1444-49.

doi:10.1097/WNR.0b013e328331a51a.

Perotto AO, "Anatomical guide for the electromyographer: the limbs and trunk." 2011; Charles C Thomas Publisher.

Richman JS, Moorman JR, "Physiological Time-Series Analysis Using Approximate Entropy and Sample Entropy." Am. J. Physiol. - Heart C. 2000; 278: H2039-49. doi:10.1152/ajpheart.2000.278.6.H2039

Rose MH, Løkkegaard A, Sonne-Holm A, Jensen BR, "Improved Clinical Status, Quality of Life, and Walking Capacity in Parkinson's Disease After Body Weight-Supported High-Intensity Locomotor Training." Arch. Phys. Med. Rehab. 2013a; 94: 687-92. doi:10.1016/j.apmr.2012.11.025.

Rose MH, Løkkegaard A, Sonne-Holm A, Jensen BR, “Tremor Irregularity, Torque Steadiness and Rate of Force Development in Parkinson's Disease.” Motor Control 2013b; 17: 203-16. doi:10.1123/mcj.17.2.203

Rose MH, Løkkegaard A, Sonne-Holm S, Jensen BR, "Effects of training and weight support on muscle activation in Parkinson's Disease." J. Electromyogr Kinesiol. 2013c; 23: 14991504.

doi:10.1016/j.jelekin.2013.07.012

Schmied A, Descarreaux M, "Reliability of EMG Determinism to Detect Changes in Motor Unit Synchrony and Coherence during Submaximal Contraction." J. Neurosci. Meth. 2011; 196: $238-46$. doi:10.1016/j.jneumeth.2011.01.005.

Scholten M, Klotz R, Plewnia C, Wächter T, Mielke C, Bloem BR, et al., "Neuromuscular Correlates of Subthalamic Stimulation and Upper Limb Freezing in Parkinson's Disease.” Clin. Neurophysiol. 2016; 127: 610-20. doi:10.1016/j.clinph.2015.02.012.

Takens F. “Detecting Strange Attractors in Turbulence," 1981; 366-81. Springer Berlin Heidelberg, 
doi:10.1007/BFb0091924.

Terry K, Griffin L, "How Computational Technique and Spike Train Properties Affect Coherence Detection." J. Neurosci. Meth. 2008; 168: 212-23. doi:10.1016/j.jneumeth.2007.09.014.

Timmermann L, Gross J, Dirks M, Volkmann J, Freund HJ, Schnitzler A, “The Cerebral Oscillatory Network of Parkinsonian Resting Tremor.” Brain 2003; 126: 199-212. doi: 10.1093/brain/awg022

Theiler J, "Spurious Dimension from Correlation Algorithms Applied to Limited Time-Series Data." Phys. Rev. A 1986; 34: 2427-32. doi:10.1103/PhysRevA.34.2427.

Vaillancourt DE, Newell KM. “The Dynamics of Resting and Postural Tremor in Parkinson's Disease." Clin. Neurophysiol. 2000; 111: 2046-56. doi:10.1016/S1388-2457(00)00467-3.

van der Stouwe AMM., Conway BA, Elting JW, Tijssen MAJ, Maurits NM, "Usefulness of Intermuscular Coherence and Cumulant Analysis in the Diagnosis of Postural Tremor." Clin. Neurophysiol. 2015; 126: 1564-69. doi:10.1016/j.clinph.2014.10.157.

Webber CL, Schmidt MA, Walsh JM, "Influence of Isometric Loading on Biceps EMG Dynamics as Assessed by Linear and Nonlinear Tools.” J. Appl. Physiol. 1995; 78: 814-22. doi:10.1152/jappl.1995.78.3.814.

Webber CL, Zbilut JP, "Dynamical Assessment of Physiological Systems and States Using Recurrence Plot Strategies.” J. Appl. Physiol. 1994; 76: 965-73. doi:10.1152/jappl.1994.76.2.965 


\section{FIGURE CAPTIONS:}

Figure 1.

A) Surface EMG of the vastus lateralis and corresponding torque signal of PD subject 11, recorded during 25s isometric contraction at $15 \%$ MVC. The data shown was recorded from the most affected leg. B) Averaged power spectrum of the torque signal in PD subjects pre-therapy and controls. Torque power is heavily concentrated below $10 \mathrm{~Hz}$. A $2 \mathrm{~Hz}$ high-pass filter was applied to the torque signal prior to calculating the power spectrum. C) Estimated coherence spectrum from rectified EMG of the vastus lateralis and vastus medialis, averaged across PD subjects pre-therapy and in controls. D) Recurrence plot (RP) corresponding to $1.5 \mathrm{~s}$ of EMG highlighted in part A. [\%REC $=36.4 \%, \% \mathrm{DET}=84.82 \%]$.

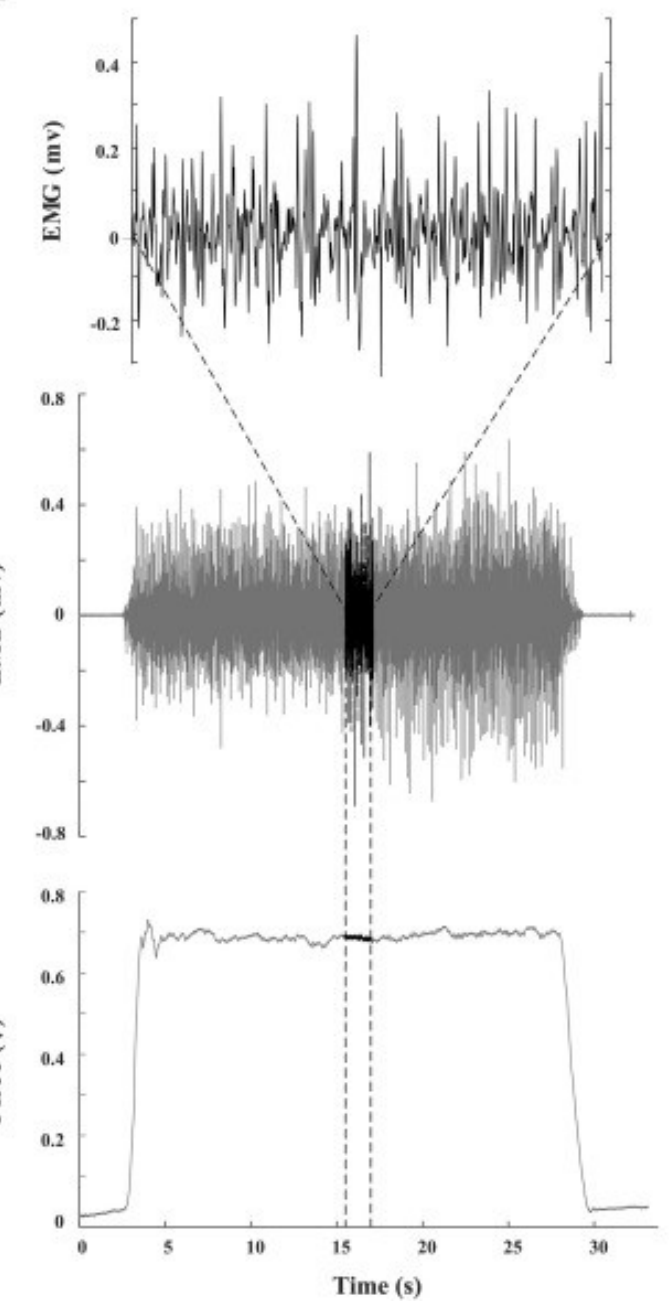

B

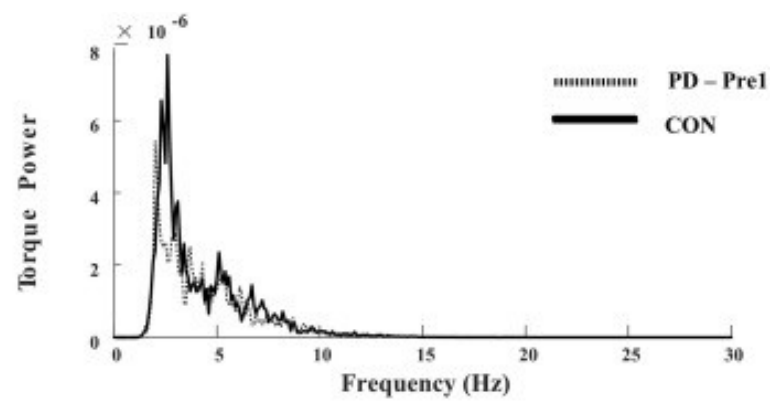

C

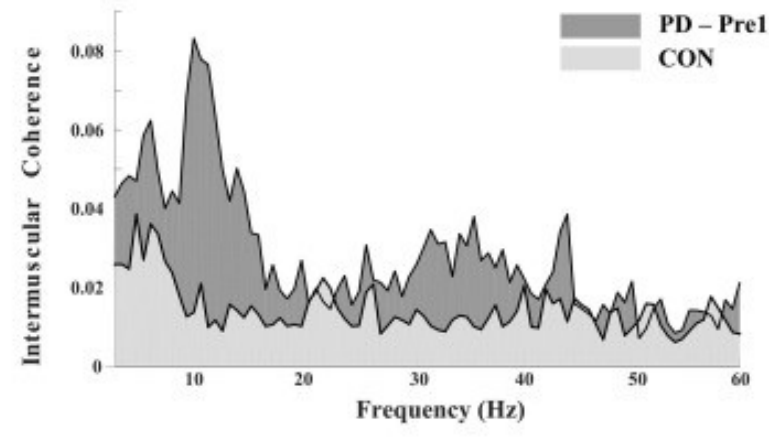

D

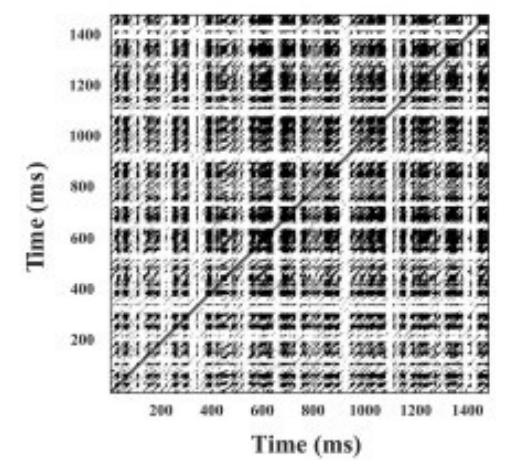


Figure 2.

Median values of \%DET and SampEn of each muscle. \%DET was consistently higher and SampEn was consistently lower in EMG of individuals with PD, with significant differences observed in the biceps femoris muscle.

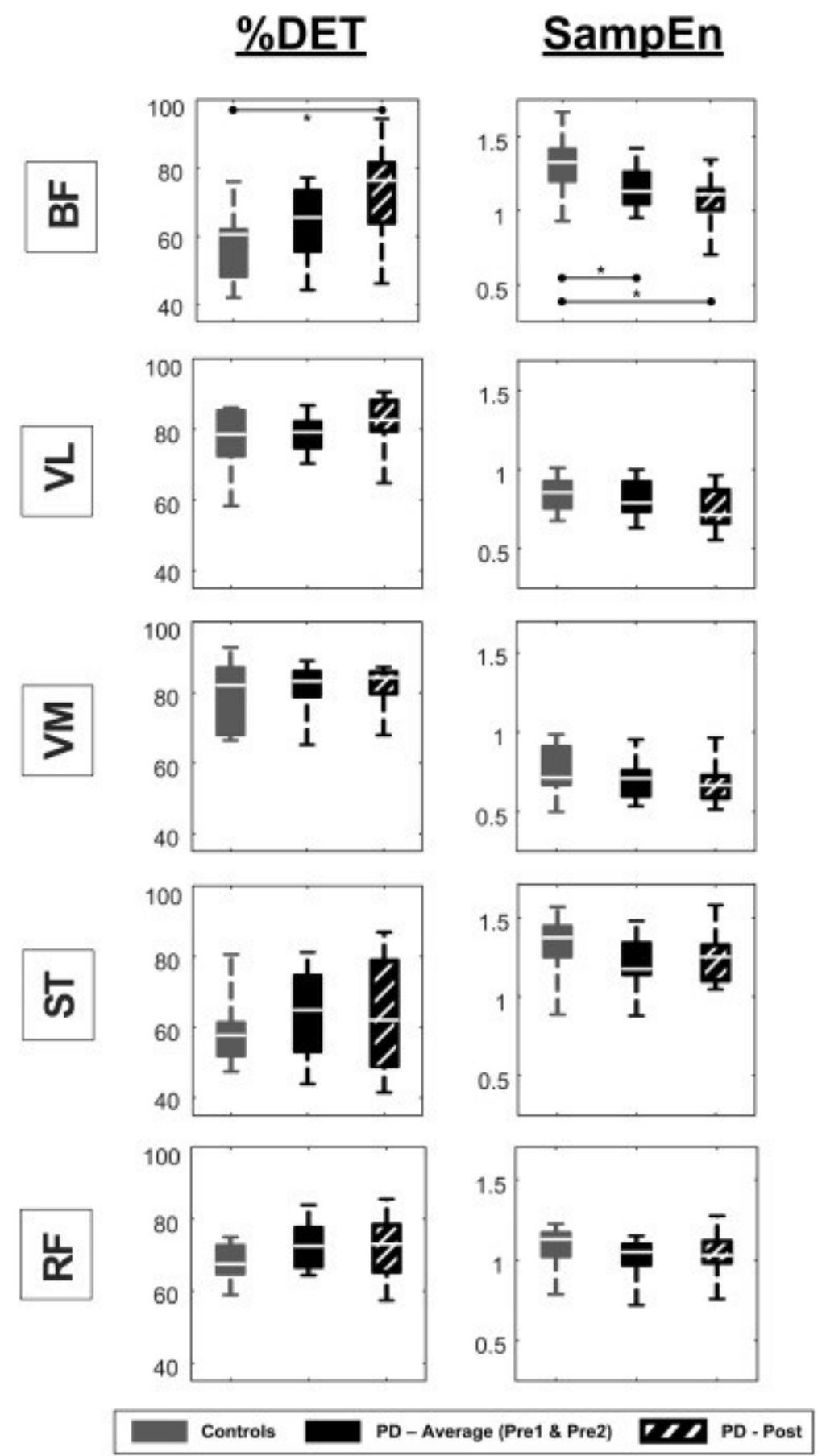


Figure 3.

Averaged \%DET and SampEn of agonist and antagonist muscles. A) Median \%DET was higher in PD subjects in pretraining and post-training stages. B) Significant differences were observed in antagonist muscles between PD patients posttraining and controls $(p=0.005)$. Median SampEn was significantly lower in PD agonist EMG post training $(p=0.021)(C)$ and antagonist EMG in both training stages (pre: $p=0.012$; post: $p=0.003)(D)$. Both nonlinear EMG features showed significant differences between agonist and antagonist muscle within subject groups $(\mathrm{p}<0.001)$, and between PD antagonist muscles pre and post training (\%DET: $\mathrm{p}=0.012$; SampEn: $\mathrm{p}=0.009)$.

A


B

Average Antagonists

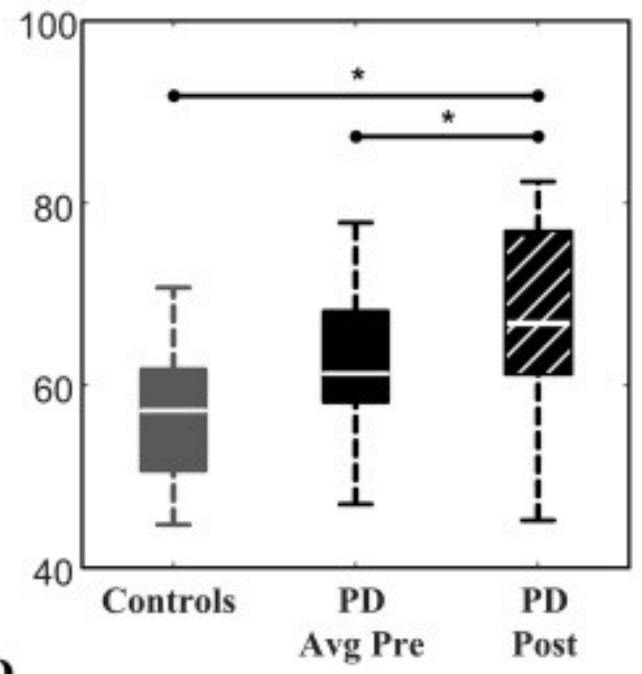

D




Figure 4.

A) Average agonist-agonist intermuscular coherence for theta, alpha, beta and low gamma frequency bands. Significant differences between subject groups were observed in the theta, alpha and beta bands $\left(\dagger \mathrm{p}=0.008, \bullet p=0.0146,{ }^{*} p=0.0137\right.$, $* * \mathrm{p}=0.0119)$. B) Average antagonist-agonist intermuscular coherence for theta, alpha, beta and low gamma frequency bands. Significant differences between subject groups were observed in the beta band post-therapy $(\mathrm{p}=0.014)$. 
A
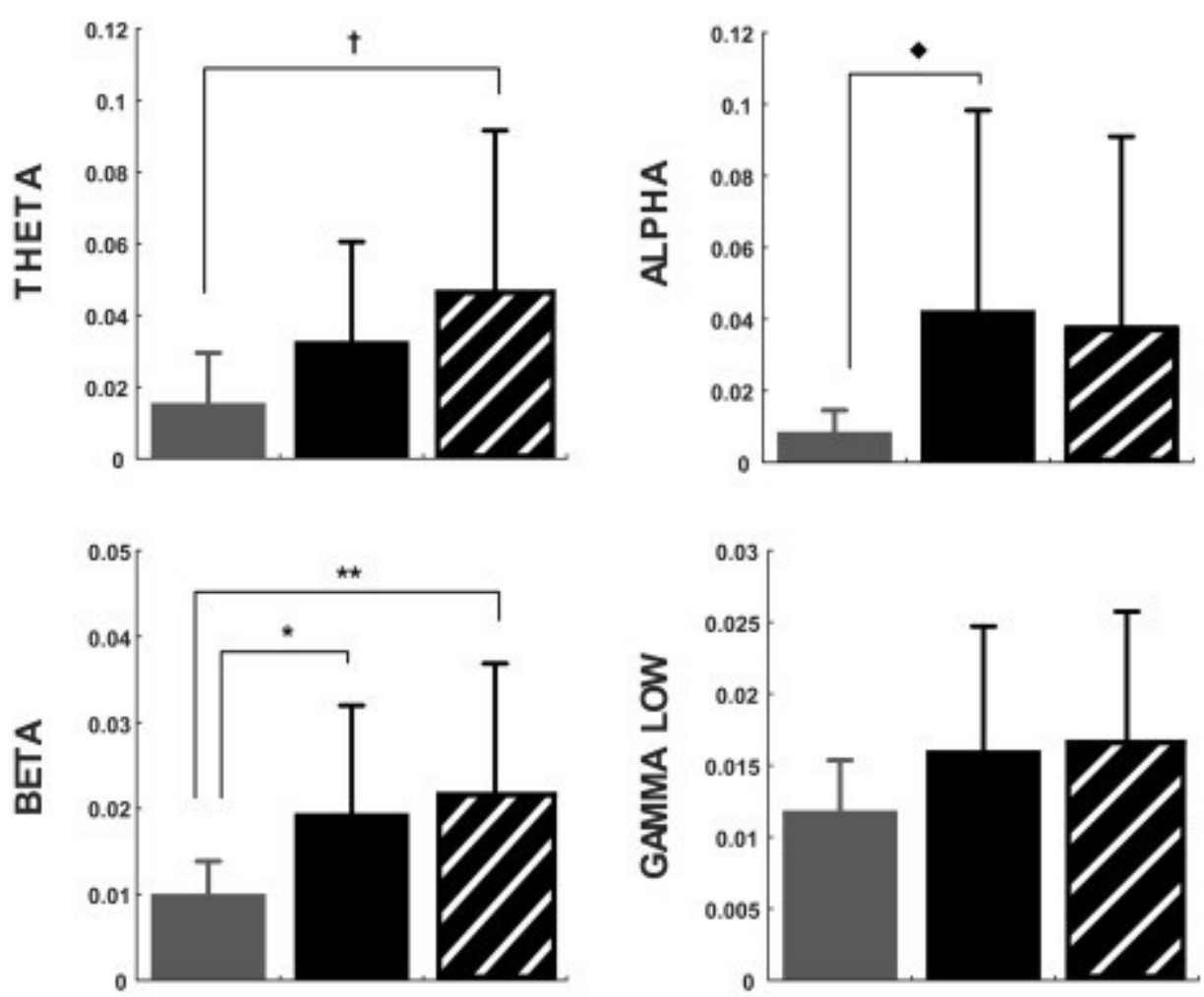

\section{B}
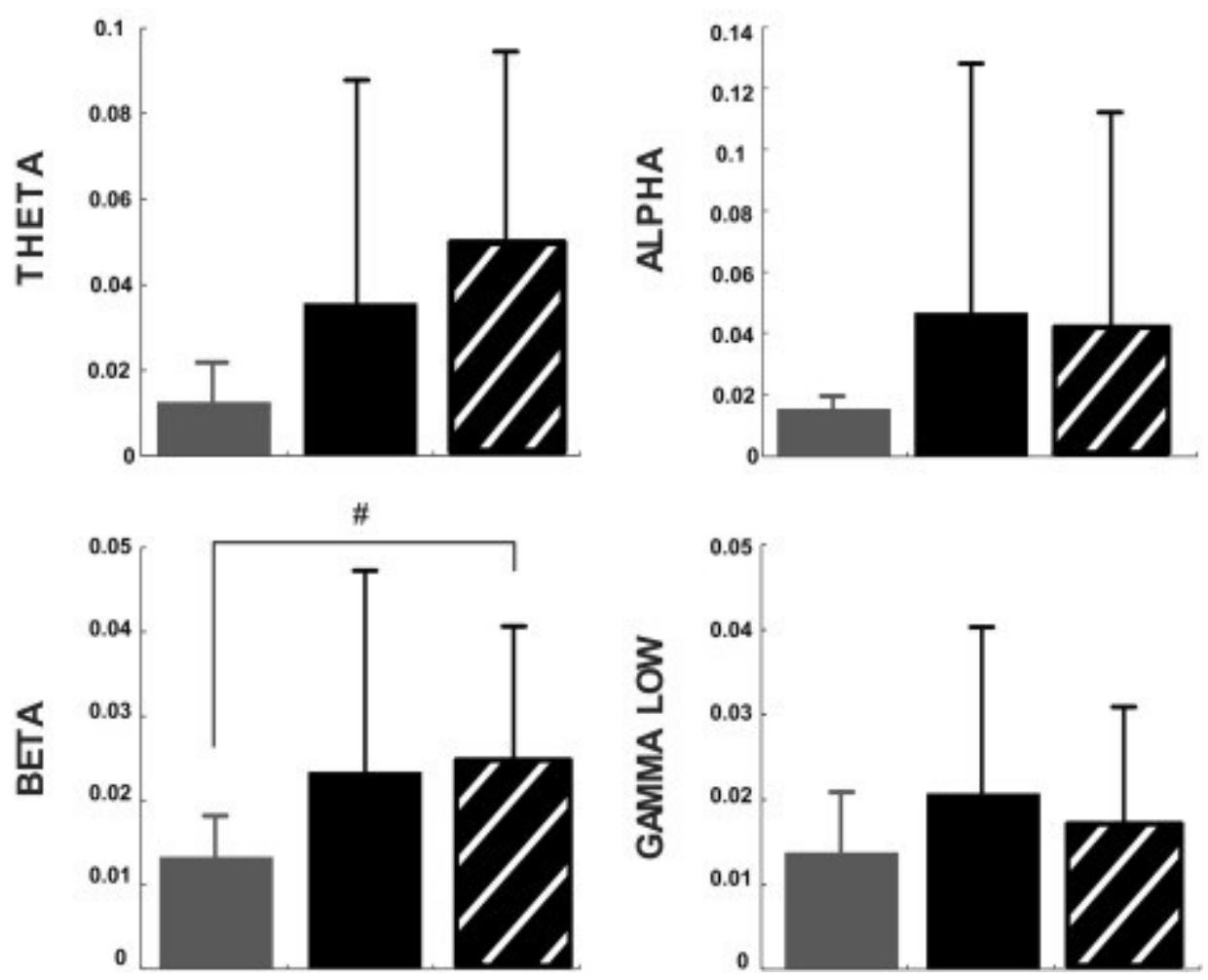
Figure 5.

Correlation between MDS-UPDRS scores and \%DET (A) and SampEn (B) at each training stage. Strong correlations were observed between both MDS-UPDRS scores and nonlinear features, in both agonist and antagonist muscles in Pre1 and Post training stages, but not in Pre2. C) Correlations between agonist-agonist theta band intermuscular coherence with MDS-

UPDRS scores for each stage of training. D) Significant correlations between theta band intermuscular coherence and variance of torque were observed in Pre1 and Post training. All correlation statistics were calculated using Spearman's rank correlation.

Pre 1

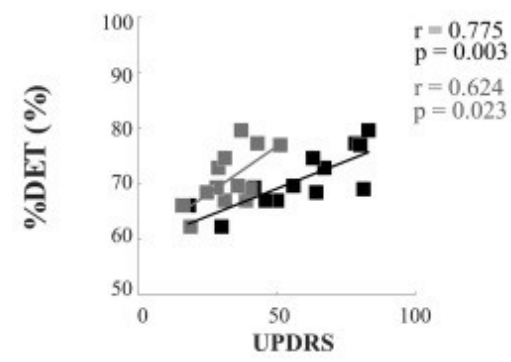

B
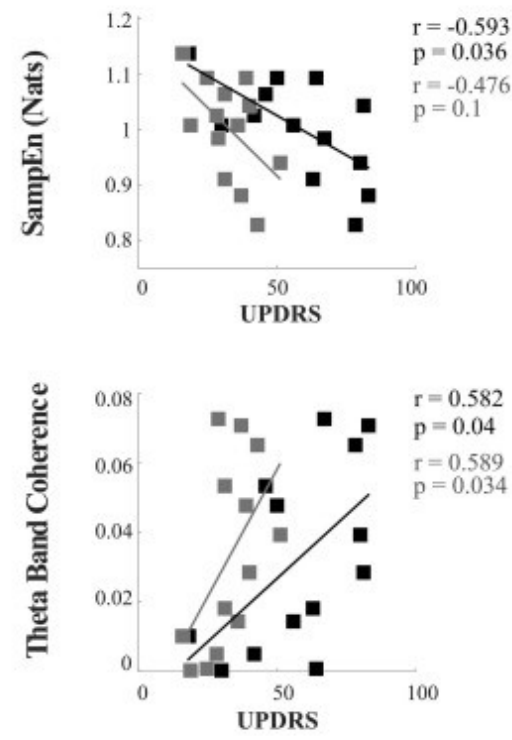

D

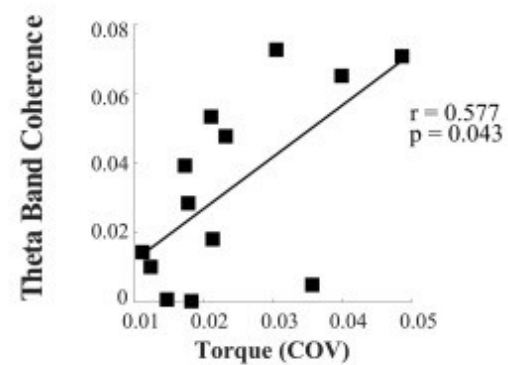

Pre 2

Post
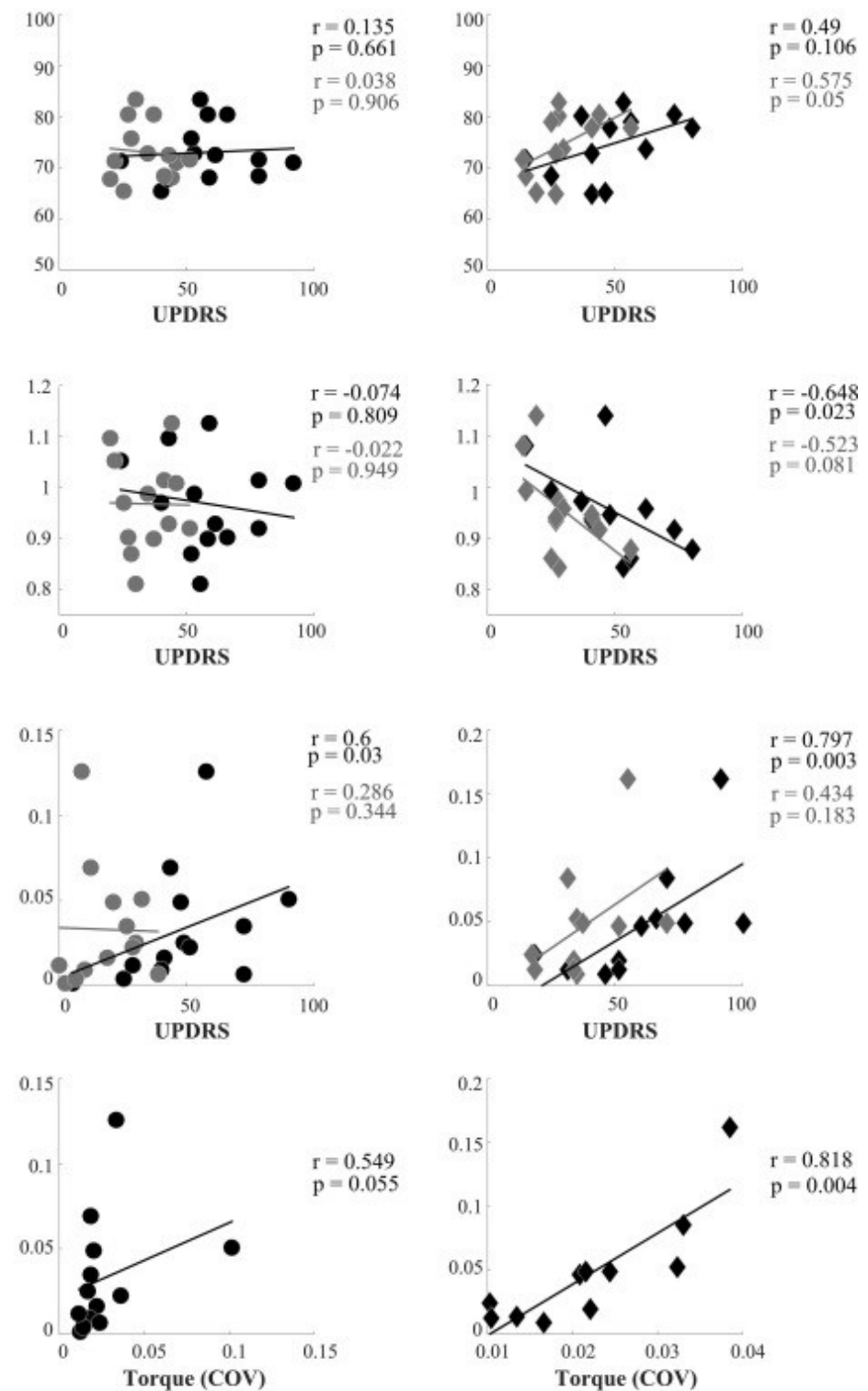

UPDRS III (Motor Score) UPDRS (Total Scere) 


\section{Tables}

Table 1.

Clinical patient profiles of all PD subjects. [HY-Hoehn \& Yahr; LED - Levodopa Equivalent Dosage]. For further information see Rose et. al. 2013a.

\begin{tabular}{|c|c|c|c|c|c|c|}
\hline Patient & Age(y) & $\begin{array}{l}\text { Disease Duration } \\
\text { (y) }\end{array}$ & HY & $\begin{array}{l}\text { MDS-UPDRS (Motor } \\
\text { Part) }\end{array}$ & $\begin{array}{l}\text { MDS- } \\
\text { UPDRS(Total) }\end{array}$ & $\begin{array}{l}\text { Medication(LED in } \\
\mathrm{mg})\end{array}$ \\
\hline 1 & 64 & 7 & 2 & 16 & 18 & 510 \\
\hline 2 & 60 & 3 & 2 & 39 & 50 & 591 \\
\hline 3 & 56 & 6 & 2 & 43 & 78 & 1163 \\
\hline 4 & 71 & 4 & 2 & 40 & 81 & 710 \\
\hline 5 & 64 & 8 & 2 & 31 & 46 & 340 \\
\hline 6 & 53 & 8 & 2 & 36 & 56 & 975 \\
\hline 7 & 64 & 5 & 2 & 29 & 67 & 620 \\
\hline 8 & 70 & 13 & 3 & 37 & 83 & 950 \\
\hline 9 & 75 & 5 & 2 & 28 & 42 & 665 \\
\hline 10 & 68 & 8 & 2 & 19 & 30 & 310 \\
\hline 11 & 58 & 1 & 2 & 51 & 80 & 160 \\
\hline 12 & 63 & 9 & 2 & 31 & 63 & 1298 \\
\hline 13 & 58 & 6 & 2 & 25 & 64 & 1563 \\
\hline
\end{tabular}


Table 2.

Parameter values used to calculate \%DET, SampEn and intermuscular coherence. The RQA embedding dimension, $\mathrm{m}$, and time delay, $\lambda$, were chosen to reflect the approximate duration of a motor unit action potential detected at the skin surface $(15 \mathrm{~ms})$ to enable repeating structures within the EMG signal to be captured. The Line parameter, 1 min, was chosen as to reflect a period of time over which the dynamics of the neuromuscular system become deterministic, comparable to the duration of a surface motor unit action. The Theiler window, $\tau$, was selected so as to remove recurrent states occurring on the same phase space trajectory.

Nonlinear Measures

\section{\%DET}

Embedding Dimension $(m)$

Delay $(\lambda)$

[\% Standard Deviation]

Radius $(\varepsilon)$

[\% Max Euclidean Distance] $\quad 20 \%$

Line $\left(I_{\min }\right)$

Theiler Window ( $T$ )
$10 \mathrm{~ms}$

Value SampEn

15

1

$20 \%$

Overlap

$75 \%$

$0 \%$

$5 \mathrm{~ms}$

Radius ( $r$ )

\section{Linear Measures}

Value Intermuscular Coherence Value

Hanning Window

$1.5 \mathrm{~s}$

$\mathrm{Nfft}$

$1.5 \mathrm{~s}$

Significance Level $(\alpha)$

0.05

Concatenated Signal Length 
Table 3. Mean \pm SD of $\% D E T$ and SampEn values of each muscle. Significant differences were observed between PD subjects and controls for both EMG features in the biceps femoris.

\begin{tabular}{|c|c|c|c|c|c|c|}
\hline \multirow[t]{2}{*}{ Muscle } & \multicolumn{3}{|l|}{$\% D E T$} & \multicolumn{3}{|c|}{ SampEn } \\
\hline & Control & $\begin{array}{l}\text { Average Pre1 + } \\
\text { Pre2 }\end{array}$ & Post & Control & $\begin{array}{l}\text { Average Pre1 + } \\
\text { Pre2 }\end{array}$ & Post \\
\hline Biceps Femoris & $56.78 \pm$ & $63.89 \pm 11.05$ & $72.37 \pm$ & $1.31 \pm$ & $1.16 \pm 0.14^{-}$ & $1.05 \pm 0.18$ \\
\hline$(B F)$ & 10.55 & & $15.6^{*}$ & 0.018 & & 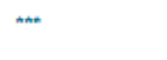 \\
\hline Vastus Lateralis & $77.78 \pm$ & $78.6 \pm 5.37$ & $82.46 \pm$ & $0.85 \pm$ & $0.82 \pm 0.12$ & $0.75 \pm 0.13$ \\
\hline$(V L)$ & 8.38 & & 7.36 & 0.01 & & \\
\hline Vastus Medialis & $78.52 \pm$ & $81.87 \pm 6.29$ & $81.52 \pm$ & $0.75 \pm$ & $0.69 \pm 0.11$ & $0.68 \pm 0.14$ \\
\hline$(V M)$ & 9.24 & & 6.41 & 0.016 & & \\
\hline Rectus Femoris & $67.9 \pm 5.43$ & $72.69 \pm 7.09$ & $72.33 \pm$ & $1.08 \pm$ & $1.02 \pm 0.13$ & $1.04 \pm 0.15$ \\
\hline$(R F)$ & & & 9.37 & 0.12 & & \\
\hline Semitendinosus & $57.47 \pm$ & $62.28 \pm 12.81$ & $64.05 \pm$ & 1.34 & $1.23 \pm 0.17$ & $1.25 \pm 0.17$ \\
\hline$(S T)$ & 8.55 & & 16.3 & \pm 0.017 & & \\
\hline \multicolumn{7}{|l|}{ * } \\
\hline \multicolumn{7}{|l|}{$p=0.0042$} \\
\hline \multicolumn{7}{|l|}{$* *$} \\
\hline \multicolumn{7}{|l|}{$p=0.016$} \\
\hline \multicolumn{7}{|l|}{$* * *$} \\
\hline$p<0.001$ & & & & & & \\
\hline
\end{tabular}

